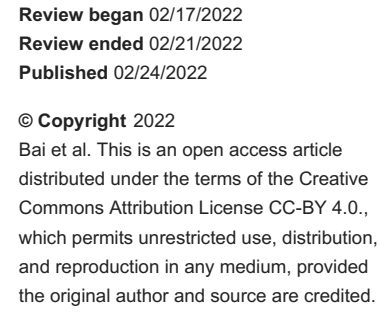

\section{Etiology, Clinical Profile, and Short-Term Outcome of Children With Acute Kidney Injury}

\author{
Shabeeta Bai ${ }^{1}$, Khemchand N. Moorani ${ }^{1}$, Bilquis Naeem ${ }^{1}$, Muhammad Ashfaq ${ }^{2}$, Rajesh . ${ }^{3}$, Ejaz Ur
} Rehman ${ }^{4}$

1. Pediatric Nephrology, National Institute of Child Health, Karachi, PAK 2. Pediatrics, National Institute of Child Health, Karachi, PAK 3. Medicine, Sir Syed College of Medical Sciences for Girls, Karachi, PAK 4. Pediatrics, United Medical and Dental College, Karachi, PAK

Corresponding author: Shabeeta Bai, shabeetaamar@gmail.com

\begin{abstract}
Background: Acute kidney injury (AKI) is a common clinical syndrome in hospitalized children and it imposes heavy burden of mortality and morbidity. In resource-constraint settings, management of AKI is very challenging and associated with adverse outcomes. The aim of this study was to determine the clinicoetiological profile and outcome of AKI.
\end{abstract}

Methodology: This prospective observational study was done at the department of pediatric nephrology and pediatric intensive care unit, National Institute of Child Health, Karachi, Pakistan from December 2020 to May 2021. A total of 130 children aged 1 month to 15 years, diagnosed with AKI irrespective of the underlying cause were included. Detailed medical information of each child including medical history, examination, and baseline investigations were obtained. Clinical and etiological profile of patients was noted. The patients were followed up to three months and the outcome was noted.

Results: In a total of 130 children, 82 (63.1\%) were male. The mean age was $5.5 \pm 4.4$ years (ranging between 1 month and 15 years). There were 117 (90.0\%) children who were referred from other centers for either dialysis or surgical treatment. Prerenal cause of AKI was found in 66 (50.8\%) children, followed by renal 53 (40.8\%) and postrenal in 11 (8.5\%) cases. Fever and shortness of breath were the most common clinical presenting symptoms in $102(78.5 \%)$ and 100 (76\%) cases, respectively. There were 45 (34.6\%) cases who were managed conservatively, 80 (61.5\%) needed dialysis, while three children were managed with plasmapheresis and two required surgical intervention in the emergency department. At threemonth follow-up period, 64 (49.2\%) children recovered (including nine with partial recovery), 46 (36.1\%) expired, 9 (6.9\%) developed end-stage renal disease, while 11 (8.5\%) had chronic kidney disease.

Conclusion: Sepsis, nephrotoxic drugs, and acute glomerulonephritis were the major causes of AKI at our center. Mortality was high among children presenting with AKI. A relatively high proportion of children with younger age, septic AKI, and presentation in critical condition could be the reasons for this high mortality.

Categories: Pediatrics, Nephrology

Keywords: end-stage renal disease, outcome, clinical profile, etiology, acute kidney injury

\section{Introduction}

Acute kidney injury (AKI) is a common clinical syndrome in hospitalized children and it imposes heavy burden of mortality and morbidity. More than 13 million people suffer from AKI each year and $85 \%$ of them live in developing countries [1]. Further, it is estimated that 1.7 million deaths occur each year from AKI and $60 \%$ of AKI survivors may experience residual renal abnormalities: proteinuria, hypertension, and reduced glomerular filtration rate (GFR) [2]. Incidence, etiology, and outcome of pediatric AKI are quite variable and depend upon age, geographic region, and clinical setting. A prospective study from India reported the prevalence of AKI in critically ill patients as $6 \%$ while development was independently linked with a significantly higher 30-day mortality rate [3]. Global data report mortality rates between $11 \%$ and $63 \%$ among children with AKI [4,5]. A study from Pakistan reported oliguria/anuria (83.6\%), hypertension (37.1\%), and severe anemia (17.2\%) to be the most common presentations among children with AKI while post-infectious glomerulonephritis (PIGN) and crescentic glomerulonephritis in primary renal, obstructive urolithiasis in postrenal, and sepsis in prerenal were the most common etiologies [6].

Differences exist regarding the most common presentation and etiologies among children with AKI, and it is necessary to analyze the presentation, etiology, and outcome at our institute. National Institute of Child Health (NICH) is one of the largest public sector hospitals in Karachi, Pakistan, where diagnostic facilities and management are provided free of cost. Neonatal intensive care unit and pediatric ICU (PICU) facilities are available, so the young children with AKI who need peritoneal dialysis are mostly referred to our center. This study was designed to prospectively determine the etiology, clinical profile, and treatment outcome of 


\section{Cureus}

AKI in children.

\section{Materials And Methods \\ Design, place, and duration of the study}

This is a prospective observational study. This study was conducted at the department of pediatric nephrology and PICU, NICH, Karachi, Pakistan from December 2020 to May 2021.

\section{Inclusion and exclusion criteria}

Children aged one month to 15 years, diagnosed with AKI irrespective of the underlying cause were included. Known cases of chronic kidney disease (CKD) or those with congenital anomalies of kidneys and urinary tract were excluded.

\section{Data collection}

Institutional Ethical Review Board (IERB) of NICH Karachi approved this study (IERB No: 40/2020). Informed and written consents were acquired from parents/guardians/caregivers of all study participants. Detailed medical information of each child including medical history, examination, and baseline investigations, i.e. complete blood count, urea, creatinine, electrolytes, urinalysis, urine, and blood cultures, and ultrasound of kidneys, ureters, and bladder were obtained. The diagnosis of AKI was based on pRIFLE (Pediatric Risk, Injury, Failure, Loss, End Stage Renal Disease) criteria [7]. Estimated GFR was determined by using Schwartz equation [8]. Further workup was done according to need in individual case like complement 3, antinuclear antibody, anti-dsDNA, and renal biopsy. All children with AKI were managed as per institutional protocol. Indications for dialysis were one or more of the following: complete anuria for $>24$ hours, volume overload, refractory metabolic acidosis, advanced uremia or uremic encephalopathy, and severe electrolyte imbalance. Peritoneal dialysis was the preferred modality in children $<10 \mathrm{~kg}$ and children with unstable hemodynamics. The patients were monitored daily with serum creatinine and urine output before, during dialysis, and till discharge. The patients were followed up to three months according to clinical status at discharge and laboratory parameters.

For each patient, predesigned proforma was filled recording to relevant details. Severity of AKI at presentation was graded using pRIFLE criteria. Etiology of AKI was grouped as prerenal, renal, and postrenal. Outcome of AKI was measured in terms of mortality, partial recovery (CKD, proteinuria, hypertension, recurrent urinary tract infection, normalization of urinary stream), and need for long-term dialysis to label as end-stage renal disease (ESRD).

\section{Statistical analysis}

SPSS version 26.0 (IBM Corp, Armonk, NY) was employed for data analysis. Qualitative data were expressed as frequency and percentages. Quantitative variables were represented as mean and standard deviation. Chisquare test was performed comparing the outcome of children as per pRIFLE criteria considering $\mathrm{P}<0.05$ as significant.

\section{Results}

In a total of 130 children, 82 (63.1\%) were male and 48 (36.9\%) were female with a male-to-female ratio of 1.7:1. The mean age was $5.5 \pm 4.4$ years (ranging between one month and 15 years). The most common age group was between 5 and 15 years accounting for $46.9 \%$ cases followed by $1-5$ years of age (29.2\%) and infants (23.8\%). Majority of the patients, 78 (60.0\%), belonged to urban areas. Poor socioeconomic status was noted in 78 (60.0\%) children. There were 117 (90.0\%) children who were referred from other centers for either dialysis or surgical treatment.

\section{Etiology of AKI}

Prerenal cause of AKI was found in 66 (50.8\%) children, followed by renal 53 (40.8\%) and postrenal in 11 (8.5\%) cases. The most common etiology in prerenal and renal AKI was infectious followed by drug-induced. Among the infectious etiologies of AKI, urosepsis was the most common, 24 (36.4\%), followed by acute gastroenteritis (AGE) in 15 (22.7\%), malaria in $5(9.4 \%)$, tetanus in 5 (7.8\%), dengue shock syndrome in 2 (3.0\%), meningococcemia in $1(1.5 \%)$, and diphtheria in $1(1.5 \%)$. Hepatorenal syndrome secondary to acute viral hepatitis was observed in 2 (3.0\%) cases. Sepsis and AGE were the most common causes of AKI in children $<5$ years. Among the intrinsic category of AKI, drug-induced AKI and hemolytic uremic syndrome were observed in 16 (30.2\%) and 13 (24.5\%), respectively. In drug-induced AKI, vancomycin was found in eight cases while captopril was found in five cases. Among the postrenal etiologies, obstructive urolithiasis was observed in nine (81.8\%) cases and two (18.2\%) had pelvi-ureteric junction obstruction. Table 1 shows the etiologies of AKI. 


\section{Cureus}

\begin{tabular}{|c|c|c|c|}
\hline \multicolumn{2}{|c|}{ Etiologies of Acute Kidney Injury } & Number & (\%) \\
\hline \multirow{11}{*}{ Prerenal $(n=66)$} & Sepsis & 24 & 36.4 \\
\hline & Acute gastroenteritis & 15 & 22.7 \\
\hline & Diabetic ketoacidosis & 8 & 12.1 \\
\hline & Tetanus & 5 & 7.8 \\
\hline & Meningococcemia & 1 & 1.5 \\
\hline & Diphtheria & 1 & 1.5 \\
\hline & Dengue shock syndrome & 2 & 3.0 \\
\hline & Hepatorenal syndrome & 2 & 3.0 \\
\hline & Congenital adrenal hyperplasia & 1 & 1.5 \\
\hline & Tumor lysis syndrome & 4 & 6.1 \\
\hline & Myocarditis & 3 & 4.5 \\
\hline \multirow{11}{*}{ Renal causes $(n=53)$} & Drug-induced & 16 & 30.2 \\
\hline & Post-infectious glomerulonephritis & 6 & 11.3 \\
\hline & Acute tubular necrosis & 2 & 3.8 \\
\hline & Membranoproliferative glomerulonephritis & 2 & 3.8 \\
\hline & Lupus nephritis & 2 & 3.8 \\
\hline & Crescentic glomerulonephritis & 2 & 3.8 \\
\hline & Rapidly progressive glomerulonephritis & 2 & 3.8 \\
\hline & Hemolytic uremic syndrome & 13 & 24.5 \\
\hline & Tubulointerstitial nephritis & 1 & 1.9 \\
\hline & Malarial nephropathy & 5 & 9.4 \\
\hline & Scorpion bite & 2 & 3.8 \\
\hline \multirow{2}{*}{ Postrenal $(n=11)$} & Stone & 9 & 81.8 \\
\hline & Pelvi-ureteric junction ob & 2 & 18.2 \\
\hline
\end{tabular}

TABLE 1: Etiology of acute kidney injuries in children

Fever and shortness of breath were the most common clinical presenting symptoms in $102(78.5 \%)$ and 100 (76\%) cases, respectively. Oliguria/anuria was noted in 69 (53.1\%) and altered sensorium in 67 (51.5\%) were also found. There were 46 (35.4\%) children who needed inotropic support. On examination, 54 (41.5\%) had signs of fluid overload and 60 (46.2\%) had signs of shock, while 24 (18.5\%) were hypertensive.

Hypernatremia was noted in 46 (35.4\%) children. Table 2 shows the clinical profile of children with AKI. 


\section{Cureus}

\begin{tabular}{|c|c|c|}
\hline Clinical Profile & $\mathbf{n}$ & $\%$ \\
\hline Fever & 102 & 78.5 \\
\hline Shortness of breath & 100 & 76.9 \\
\hline Oliguria/anuria & 69 & 53.1 \\
\hline Altered sensorium & 67 & 51.5 \\
\hline Loose motion & 43 & 33.1 \\
\hline Vomiting & 62 & 47.7 \\
\hline Sign of fluid overload & 54 & 41.5 \\
\hline Inotropic support & 46 & 35.4 \\
\hline Signs of shock & 60 & 46.2 \\
\hline Blood Pressure $>95$ th centile & 24 & 18.5 \\
\hline Anemia & 68 & 52.3 \\
\hline Dehydration & 24 & 18.5 \\
\hline Rashes & 9 & 6.9 \\
\hline Leucocyte count $>15,000$ & 63 & 47.7 \\
\hline Thrombocytopenia & 40 & 30.8 \\
\hline Hypernatremia & 46 & 35.5 \\
\hline Echogenic kidneys on ultrasonography & 27 & 20.7 \\
\hline
\end{tabular}

TABLE 2: Clinical and laboratory features of children with acute kidney injury

\section{Severity of AKI at presentation}

As per pRIFLE criteria, the majority of children were in failure category, 119 (91.5\%), at presentation, 9 (6.9\%) were in injury, and $2(1.5 \%)$ in risk category.

\section{Outcome of children with AKI}

There were 45 (34.6\%) cases who were managed conservatively, 80 (61.5\%) needed dialysis (44 needed peritoneal dialysis and 36 hemodialysis), while three children managed with plasmapheresis and two required surgical intervention in the emergency department. At three-month follow-up period, 64 (49.2\%) children recovered (including nine with partial recovery), 46 (36.1\%) expired, 9 (6.9\%) developed ESRD, while 11 (8.5\%) had CKD. Table 3 shows the details of outcome according to pRIFLE criteria at threemonth follow-up.

\begin{tabular}{|c|c|c|c|c|c|}
\hline pRIFLE Categories & Recovered & CKD & ESRD & Death & P-Value \\
\hline Risk(n=2) & 1 & - & - & 1 & \multirow{3}{*}{0.6247} \\
\hline Injury (n=9) & 7 & 1 & - & 1 & \\
\hline Failure $(n=119)$ & 56 & 10 & 9 & 44 & \\
\hline
\end{tabular}

\section{TABLE 3: Outcome according to pRIFLE criteria at three-month follow-up}

CKD: chronic kidney disease; ESRD: end-stage renal disease; pRIFLE: Pediatric Risk, Injury, Failure, Loss, End-Stage Renal Disease

\section{Discussion}

AKI contributes to high childhood morbidity and mortality and increases significant economic burden on 
the family and healthcare system [9]. Early identification of risk factors can prevent the initiation of disease and enhance prompt diagnosis and treatment [10]. There is very limited local data regarding risk factors and outcome of AKI especially in children $<5$ years. AKI in the pediatric age group is associated with various etiologies. Etiology and epidemiology of AKI may vary from center to center within a same country. In our cohort of 130 children with AKI, $63 \%$ were male, resulting in a male-to-female ratio of $1.7: 1$, which is quite similar to the ratio of other developing countries [11,12]. In this study, a majority (91.4\%) of the children were in failure category at the time of presentation with GFR $<35 \mathrm{~mL} / \mathrm{min} / 1.73 \mathrm{~m}^{2}$ as per pRIFLE criteria. This presentation with severe disease has been reported from low-income countries [12]. Possible reasons could be lack of early diagnosis, late referral, and financial constraint, which is the major concern in Pakistan where majority of people are poor and come to hospital when the child has developed severe disease.

After reviewing literature on AKI we found wide variation in etiology between developed and developing countries. In developing countries community-acquired AKI is the common cause, whereas it is hospitalacquired AKI in developed countries. This study found that the prerenal etiology of AKI was noted in $50.8 \%$, renal in $40.8 \%$, and postrenal in $8.5 \%$. A prospective study from China reported prerenal causes of AKI among 27.7\% cases of AKI [13]. Researchers have reported that the most common cause of AKI in children in developing countries are infections [14]. We reported sepsis and AGE to be the predominant etiologies behind AKI. Data from developed countries have labeled sepsis and AGE as the most common etiologies of AKI $[12,15]$. This could be due to poor hygiene and lack of sanitation.

A study from Nigeria reported that $75.8 \%$ of children had stage 3 AKI at the time of hospitalization. Sepsis (41.8\%) and primary kidney disease (29.7\%) were the most common causes [16]. In a study published in 2016 from Karachi, the results were different from our study where PIGN and urolithiasis were the most common etiologies behind AKI [6]. Exposure to nephrotoxic drugs has been recognized as a risk factor for pediatric AKI [17]. In our cohort, exposure to nephrotoxic drugs was the most common renal cause of AKI. Notably, vancomycin and captopril were the most important agents.

The outcome of AKI is dependent upon factors like age, etiology, and the time the child presented to the healthcare facility [18]. Studies from India have reported higher rates of mortality among children with AKI ranging from $28.5 \%$ to $46.3 \%$, whereas researchers from sub-Saharan Africa reported high rates of mortality as well $(34 \%)[19,20]$. In our study, mortality rate was high (35.4\%) comparatively similar to that reported from other developing countries. A relatively high proportion of children with younger age, septic AKI, and presentation in critical condition could be the reasons for this high mortality.

\section{Limitations}

First, data in this study is from a single pediatric nephrology center; therefore, it does not truly reflect the etiological spectrum of pediatric AKI prevalent throughout the country. Secondly, neonates were excluded from this study since their susceptibility and etiology of AKI are considerably different from older infants and children. Post-cardiac surgery children were not included in this study. Nevertheless, this prospective study describes very relevant information on pediatric AKI spectrum encountered at a large tertiary care referral center in a developing country.

\section{Conclusions}

Sepsis, nephrotoxic drugs, and acute glomerulonephritis were the major causes of AKI at our center. Mortality was high among children presenting with AKI. A relatively high proportion of children with younger age, septic AKI, and presentation in critical condition could be the reasons for this high mortality.

\section{Additional Information \\ Disclosures}

Human subjects: Consent was obtained or waived by all participants in this study. Institutional Ethical Review Board (IERB) issued approval 40/2020. Institutional Ethical Review Board (IERB) of National Institute of Child Health Karachi Pakistan approved this study. Animal subjects: All authors have confirmed that this study did not involve animal subjects or tissue. Conflicts of interest: In compliance with the ICMJE uniform disclosure form, all authors declare the following: Payment/services info: All authors have declared that no financial support was received from any organization for the submitted work. Financial relationships: All authors have declared that they have no financial relationships at present or within the previous three years with any organizations that might have an interest in the submitted work. Other relationships: All authors have declared that there are no other relationships or activities that could appear to have influenced the submitted work.

\section{Acknowledgements}

The authors are thankful to Muhammad Aamir Latif (Res\&Tech Institute, Bahawalpur, Pakistan) for his volunteer assistance in statistical analysis of this research. 


\section{References}

1. Lameire NH, Bagga A, Cruz D, et al.: Acute kidney injury: an increasing global concern . Lancet. 2013, 382:170-9. 10.1016/S0140-6736(13)60647-9

2. Mehta RL, Cerdá J, Burdmann EA, et al.: International Society of Nephrology's 0by25 initiative for acute kidney injury (zero preventable deaths by 2025): a human rights case for nephrology. Lancet. 2015, 385:2616-43. 10.1016/S0140-6736(15)60126-X

3. Podoll AS, Kozar R, Holcomb JB, Finkel KW: Incidence and outcome of early acute kidney injury in criticallyill trauma patients. PLoS One. 2013, 8:e77376. 10.1371/journal.pone.0077376

4. Cleto-Yamane TL, Gomes CL, Suassuna JH, Nogueira PK: Acute kidney injury epidemiology in pediatrics . J Bras Nefrol. 2019, 41:275-83. 10.1590/2175-8239-JBN-2018-0127

5. Andreoli SP: Acute kidney injury in children . Pediatr Nephrol. 2009, 24:253-63. 10.1007/s00467-008-1074-9

6. Tresa V, Yaseen A, Lanewala AA, Hashmi S, Khatri S, Ali I, Mubarak M: Etiology, clinical profile and shortterm outcome of acute kidney injury in children at a tertiary care pediatric nephrology center in Pakistan. Ren Fail. 2017, 39:26-31. 10.1080/0886022X.2016.1244074

7. Soler YA, Nieves-Plaza M, Prieto M, García-De Jesús R, Suárez-Rivera M: Pediatric Risk, Injury, Failure, Loss, End-Stage renal disease score identifies acute kidney injury and predicts mortality in critically ill children: a prospective study. Pediatr Crit Care Med. 2013, 14:e189-95. 10.1097/PCC.0b013e3182745675

8. Schwartz GJ, Muñoz A, Schneider MF, Mak RH, Kaskel F, Warady BA, Furth SL: New equations to estimate GFR in children with CKD. J Am Soc Nephrol. 2009, 20:629-37. 10.1681/ASN.2008030287

9. Silver SA, Chertow GM: The economic consequences of acute kidney injury . Nephron. 2017, 137:297-301. 10.1159/000475607

10. Finlay S, Bray B, Lewington AJ, Hunter-Rowe CT, Banerjee A, Atkinson JM, Jones MC: Identification of risk factors associated with acute kidney injury in patients admitted to acute medical units. Clin Med (Lond). 2013, 13:233-8. 10.7861/clinmedicine.13-3-233

11. Anigilaje EA, Adebayo AI, Ocheni SA: Acute kidney injury in children: a study of etiology, clinical profile, and short-term outcomes at the University of Abuja Teaching Hospital, Gwagwalada, Abuja, Nigeria. Saudi J Kidney Dis Transpl. 2019, 30:421-39. 10.4103/1319-2442.256849

12. Parikh AC, Tullu MS: A study of acute kidney injury in a tertiary care pediatric intensive care unit . J Pediatr Intensive Care. 2021, 10:264-70. 10.1055/s-0040-1716577

13. Teo SH, Lee KG, Koniman R, et al.: A prospective study of clinical characteristics and outcomes of acute kidney injury in a tertiary care Centre. BMC Nephrol. 2019, 20:282. 10.1186/s12882-019-1466-z

14. Shahrin L, Sarmin M, Rahman AS, et al.: Clinical and laboratory characteristics of acute kidney injury in infants with diarrhea: a cross-sectional study in Bangladesh. J Int Med Res. 2020, 48:300060519896913. 10.1177/0300060519896913

15. Esezobor CI, Ladapo TA, Osinaike B, Lesi FE: Paediatric acute kidney injury in a tertiary hospital in Nigeria: prevalence, causes and mortality rate. PLoS One. 2012, 7 :e51229. 10.1371/journal.pone.0051229

16. Esezobor CI, Ladapo TA, Lesi FE: Clinical profile and hospital outcome of children with severe acute kidney injury in a developing country. J Trop Pediatr. 2015, 61:54-60. 10.1093/tropej/fmu066

17. Patzer L: Nephrotoxicity as a cause of acute kidney injury in children . Pediatr Nephrol. 2008, 23:2159-73. 10.1007/s00467-007-0721-X

18. Uber AM, Sutherland SM: Acute kidney injury in hospitalized children: consequences and outcomes . Pediatr Nephrol. 2020, 35:213-20. 10.1007/s00467-018-4128-7

19. Krishnamurthy S, Narayanan P, Prabha S, Mondal N, Mahadevan S, Biswal N, Srinivasan S: Clinical profile of acute kidney injury in a pediatric intensive care unit from Southern India: a prospective observational study. Indian J Crit Care Med. 2013, 17:207-13. 10.4103/0972-5229.118412

20. Batte A, Starr MC, Schwaderer AL, et al.: Methods to estimate baseline creatinine and define acute kidney injury in lean Ugandan children with severe malaria: a prospective cohort study. BMC Nephrol. 2020, 21:417. 10.1186/s12882-020-02076-1 